\title{
Rapidly rotating Rayleigh-Bénard convection with a tilted axis
}

\author{
L. Novi* \\ Institute of Geosciences and Earth Resources (IGG), National Research Council (CNR), Pisa, 56124, Italy \\ J. von Hardenberg \\ Institute of Atmospheric Sciences and Climate (ISAC), National Research Council (CNR), Torino, 10133, Italy \\ D. W. Hughes \\ Department of Applied Mathematics, University of Leeds, Leeds LS2 9JT, United Kingdom \\ A. Provenzale \\ Institute of Geosciences and Earth Resources (IGG), National Research Council (CNR), Pisa, 56124, Italy \\ E. A. Spiegel \\ Department of Astronomy, Columbia University, 550 W 120th St, New York, New York 10027, USA
}

(Received 6 January 2019; published 28 May 2019)

\begin{abstract}
We numerically explore the dynamics of an incompressible fluid heated from below, bounded by free-slip horizontal plates and periodic lateral boundary conditions, subject to rapid rotation about a distant axis that is tilted with respect to the gravity vector. The angle $\phi$ between the rotation axis and the horizontal plane measures the tilting of the rotation axis; it can be taken as a proxy for latitude if we think of a local Cartesian representation of the convective dynamics in a rotating fluid shell. The results of the simulations indicate the existence of three different convective regimes, depending on the value of $\phi$ : (1) sheared, intermittent large-scale winds in the direction perpendicular to the plane defined by the gravity and rotation vectors, when rotation is "horizontal" $\left(\phi=0^{\circ}\right)$; (2) a large-scale cyclonic vortex tilted along the rotation axis, when the angle between the rotation axis and the gravity vector is relatively small ( $\phi$ between about $45^{\circ}$ and $\left.90^{\circ}\right)$; and (3) a new intermediate regime characterized by vertically sheared large-scale winds perpendicular to both gravity and rotation. In this regime, the winds are organized in bands that are tilted along the rotation axis, with unit horizontal wave number in the plane defined by gravity and rotation at values of $\phi$ less than about $60^{\circ}$. This intermediate solution, studied for the first time in this work, is characterized by weaker vertical heat transport than the cases with large-scale vortices. For intermediate values of $\phi$ (between about $45^{\circ}$ and $60^{\circ}$ ), the banded, sheared solution coexists with the large-scale vortex solution, with different initial conditions leading to one or the other dynamical behavior. A discussion of the possible implications of these results for the dynamics of rapidly rotating planetary atmospheres is provided.
\end{abstract}

DOI: 10.1103/PhysRevE.99.053116

\section{INTRODUCTION}

Turbulent buoyancy-driven convection is at the core of heat transport mechanisms in the atmospheres of planets, cool stars, and other celestial bodies. The main physical processes underlying turbulent convection have been widely explored over many years (e.g., Refs. [1,2]), often using the simplified setting of Rayleigh-Bénard convection (RBC) of a Boussinesq fluid as the essential model to study a layer of fluid cooled from above and/or heated from below [3]. However, in more realistic conditions, RBC is often coupled with system rotation, which can significantly alter the dynamics [4-8]. The interest in rotating convection as a recurring process in many natural systems, such as rapidly rotating astrophysical bodies,

\footnotetext{
*1juba.novi@igg.cnr.it; previously at Istituto Nazionale di Oceanografia e Geofisica Sperimentale (OGS), Borgo Grotta Gigante 42/C, 34010 Sgonico, Trieste, Italy.
}

has motivated both laboratory experiments [9-12] and numerical simulations [13-17]. The current observations of the complex structure of Jupiter's fluid motions, including jets and large-scale winds at low to midlatitudes and intense vortices at the poles [18], have renewed interest in the interplay of rotation and convection.

There are various complementary approaches to the computational modeling of convection in rotating bodies. One is to employ global models, in which a full spherical (or spherical shell) geometry is considered. At the other extreme, in some sense, are local plane layer models, which treat the dynamics in a Cartesian geometry, representative of a layer of fluid at a particular latitude; this is similar to the "f-plane" approach for geophysical flows [19]. There are also models that exploit the fact that the strong Coriolis force tends to reduce the dependence on the coordinate along the rotation axis, thus making the problem quasitwo-dimensional; for example, the annulus model developed 
by Busse [20,21] takes advantage of this feature. Each approach has its advantages and drawbacks, and each is of importance in obtaining a full understanding of turbulent convection in geophysical and astrophysical flows. Clearly, only global models can capture dynamical behavior on scales comparable to the body itself, for which the influence of spherical geometry is critical. Conversely, for computational reasons, models with a simpler geometry permit the study of more turbulent parameter regimes, closer to those attained in planetary bodies.

Recent high-resolution numerical studies of turbulent rotating RBC in a plane layer have investigated the two limiting cases in which the rotation axis is either parallel or perpendicular to gravity - what we shall refer to as "vertical" and "horizontal" rotation. The influence of vertical rotation, which models the polar regions, is to cause mergers of the turbulent convective plumes [6]; in certain parameter regimes, such mergers lead to the generation of large-scale, long-lived, coherent cyclonic vortices [22,23]. It is of interest to note that in an asymptotically reduced model, valid in the limit of the Rossby number (a measure of inertial to Coriolis forces) tending to zero, there is no distinction between cyclonic and anticyclonic motions; in this case, large-scale vortices of both signs are formed [24]. The influence of vertical rotation on the convective heat flux is intriguing; in certain regimes, heat transport is suppressed by rotation [22], whereas in others it is enhanced [6,24].

Background rotation about a horizontal axis breaks the horizontal isotropy. The consequent generation of large-scale winds (perpendicular to both rotation and gravity) can inhibit the average vertical heat flux [7], and the resulting dynamics resembles the flow in equatorial areas of rotating spherical shells [25-27].

It is important to ask how the results from local polar and equatorial models relate to those from global (spherical or spherical shell) models. Rotating Boussinesq convection in a spherical geometry has been extensively studied (e.g, Refs. [25,28]) (see the monograph [29] for a full discussion and references). The curved boundaries have an important effect on large-scale structures; in flows with high Reynolds numbers and low Rossby numbers they are, notably, responsible for the formation of zonal flows of amplitude large compared with the typical convective velocity (e.g., Ref. [30]). Unlike in local polar models, the formation of vortices at scales larger than the typical convective size has not been observed in simulations of rotating convection in spherical geometry. In a Cartesian domain, the size of the large-scale vortices may be limited only by the domain size, so that if the upscale energy transfer were allowed to continue, the vortices would eventually feel the latitudinal variation of the Coriolis parameter. In this case, it is conjectured [24] that the large-scale dynamics would become organised into zonal flows. That said, it is, however, particularly noteworthy that coherent polar vortices-reminiscent of those appearing in local polar models - have been observed on both Saturn and Jupiter.

Given the very different nature of the convective flows for the investigated cases of vertical and horizontal rotation, it is therefore of considerable interest to investigate the nature of plane layer convection when the rotation axis and the gravity vector are neither parallel nor perpendicular. The problem of the linear stability of convection in layers with tilted rotation vectors was first addressed in Ref. [31], who determined the nature of the convective motions at onset, with consideration also of the role of a thermal wind; the linear problem has also been re-examined recently in Ref. [32]. In an early pioneering numerical study, the nonlinear evolution of convection with tilted rotation was studied in Ref. [33], which explored how heat transport and mean flow generation were influenced by the tilt of the rotation vector. However, a characterization of turbulent, three-dimensional RBC at generic latitudes and relatively high Rayleigh number still remains largely unaddressed. The inherent importance of convective processes in a wide range of intermediate latitudes in rotating astrophysical and geophysical bodies further motivates the interest in filling this gap. The current work represents one step in this direction.

\section{MODEL EQUATIONS AND NUMERICAL PROCEDURE}

We use the Boussinesq approximation [4,34], with constant kinematic viscosity $v$, thermal expansion coefficient $\alpha$, thermal diffusivity $\chi$, and gravitational acceleration $g$. The simulation domain is a box with depth $d$, square horizontal section of side $L=2 \pi d$, and horizontally periodic boundary conditions in the $x$ and $y$ directions. As natural units to make the equations nondimensional we choose the domain thickness $d$, the vertical temperature difference between the bounding plates $\Delta$, and the thermal time $\tau_{\text {th }}=d^{2} / \chi$. The relative importance of buoyancy forces and viscous drag is given by the Rayleigh number $R=\left(\tau_{\text {diss }} / \tau_{\text {dyn }}\right)^{2}$, where $\tau_{\text {diss }}=\sqrt{\tau_{\mathrm{th}} \tau_{\text {vis }}}$ defines an effective dissipative time, $\tau_{\text {dyn }}=\sqrt{d /(g \alpha \Delta)}$ is the dynamical time, and $\tau_{\text {vis }}=d^{2} / \nu$ denotes the viscous time. The competition between the two dissipative terms is measured by the Prandtl number $\sigma=\tau_{\text {th }} / \tau_{\text {vis }}$. In keeping with our previous studies and the value for the atmosphere of our planet, in this study we fix $\sigma=0.71$. The nondimensional vertical domain is fixed as $-1 / 2 \leqslant z \leqslant 1 / 2$ and the horizontal coordinates range in the interval $0 \leqslant x, y \leqslant 2 \pi$. This setup is similar to that used in other previous RBC studies in the absence of rotation [35,36] and for the case of horizontal rotation [7].

The system experiences a background rotation about a distant axis with dimensional angular speed $\Omega / \tau_{\text {th }}$. The nondimensional angular speed $\Omega$ is related to the Rossby number defined in Ref. [6] by Ro $=(\sqrt{\sigma \mathrm{Ra}}) /(2 \Omega)$, and to the Ekman number defined in Ref. [22] by Ek $=\sigma /(2 \Omega)$. The rotation axis lies in the $y-z$ plane and is tilted by an angle $\phi$ with respect to the horizontal. Thus, the angle between the rotation axis and the gravity vector is $90^{\circ}-\phi$.

The nondimensional Boussinesq equations governing velocity $\boldsymbol{u}=(u, v, w)$, temperature $T$, and pressure $p$ are as follows:

$$
\begin{gathered}
\nabla \cdot \boldsymbol{u}=0, \\
\partial_{t} \boldsymbol{u}+\boldsymbol{u} \cdot \nabla \boldsymbol{u}+2 \Omega \hat{\boldsymbol{k}} \times \boldsymbol{u}=-\nabla p+\sigma R T \hat{z}+\sigma \nabla^{2} \boldsymbol{u}, \\
\partial_{t} T+\boldsymbol{u} \cdot \nabla T=\nabla^{2} T,
\end{gathered}
$$


where $\hat{\boldsymbol{k}}$ is a unit vector along the rotation axis and $\hat{z}$ is the vertical unit vector, antiparallel to gravity. The initial conditions consist of a random perturbation to the rest state. Free slip boundary conditions are imposed on the velocity at the top and bottom boundaries, where the temperature is prescribed as fixed and horizontally uniform:

$$
\begin{gathered}
\left.\frac{\partial u}{\partial z}\right|_{z=-\frac{1}{2}, \frac{1}{2}}=\left.\frac{\partial v}{\partial z}\right|_{z=-\frac{1}{2}, \frac{1}{2}}=\left.w\right|_{z=-\frac{1}{2}, \frac{1}{2}}=0 \\
\left.T\right|_{z=-\frac{1}{2}}=0.5,\left.\quad T\right|_{z=\frac{1}{2}}=-0.5 .
\end{gathered}
$$

Equations (1)-(3) are integrated numerically using a pseudospectral method in the horizontal directions with $2 / 3$ dealiasing, while second-order finite differences are used in the vertical. A third-order fractional step method is adopted in time [37]. We use a resolution of $256^{2}$ grid points in the horizontal and 192 vertical levels, spaced more densely close to the top and bottom plates in order to better resolve the boundary layers. Numerical parallelization is performed by a vertical partitioning in slices, using the MPI paradigm.

In our simulations we set $2 \Omega=10^{4}$ in nondimensional units and set the Rayleigh number at $R=10^{7}$. We vary the orientation of the rotation axis by changing $\phi$ in the range $0^{\circ} \leqslant \phi \leqslant 90^{\circ}$. Specifically, we explore the values $\phi=$ $0^{\circ}, 15^{\circ}, 30^{\circ}, 45^{\circ}, 60^{\circ}, 90^{\circ}$. This is roughly equivalent to exploring different latitudes from the equator $\left(\phi=0^{\circ}\right)$ to the pole $\left(\phi=90^{\circ}\right)$. In this analogy, by fixing Rayleigh and Ekman numbers and varying latitude, the kinetic energy and hence the Rossby number can change from case to case, thus varying the degree of supercriticality (see, e.g., Ref. [4]). Our choice of system parameters $(R, \sigma, \Omega$, fluid depth) leads to a Rossby deformation radius consistent with a fast rotation, i.e., a Rossby deformation radius smaller than the fluid depth; this is the regime on which this work focuses. Cases with a slower rotation and/or a shallower fluid layer such that the Rossby deformation radius is much larger than the fluid depth are clearly not covered in this work.

\section{RESULTS AND DISCUSSION}

The first evidence of the role played by tilted rotation in altering the convective dynamics is provided by the temporal evolution of the Nusselt number, $\mathrm{Nu}(t)=1+\left\langle w T^{\prime}\right\rangle$, where $\langle\ldots\rangle$ is a volume average over the entire domain and $T^{\prime}=$ $T-\bar{T}$ is the fluctuation of $T$ with respect to the horizontal average (denoted by the overbar). The Nusselt number is an integral quantity of the flow serving as an indicator of vertical heat transport: For diffusive transport, $\mathrm{Nu}=1$, while turbulent convective transport leads to $\mathrm{Nu} \gg 1$.

Changing the angle $\phi$ results in different temporal evolutions of the Nusselt number, as shown in Fig. 1, where three regimes can be identified after the initial transient: (1) Intermittent behavior at $\phi=0^{\circ}$, with an average value of $\mathrm{Nu}=7.4$ computed from $t=0.7$ to $t=1$. For $\phi=0^{\circ}$, the lower heat flux is associated with the onset of a wind with zero horizontal wave number, followed by intermittent behavior of the heat transport, as found in previous works $[7,38]$ and referred to as "windy convection." (2) A lower-Nu regime, significantly less intermittent, for $\phi=15^{\circ}$ and, at late times, for $\phi=30^{\circ}$, with

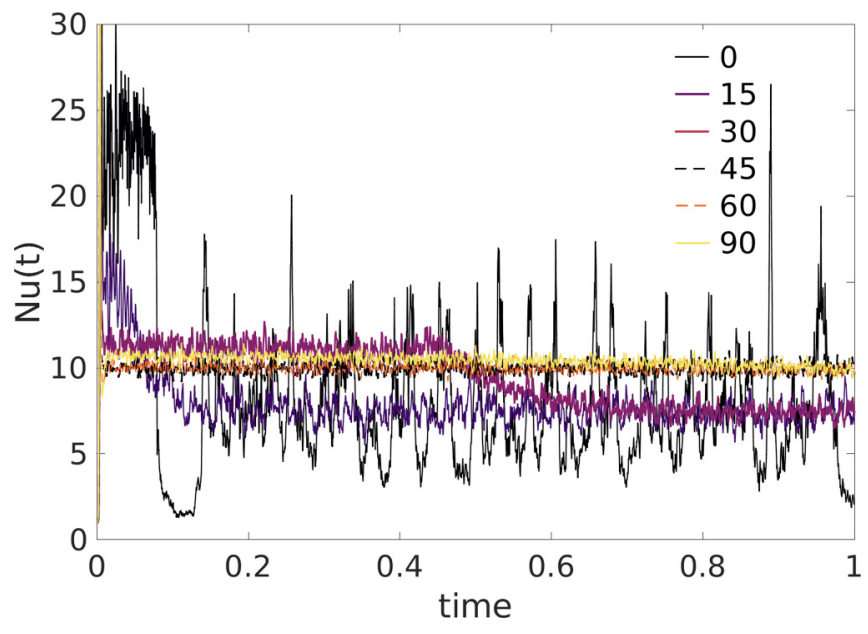

FIG. 1. Time series of the Nusselt number for all simulations. Each line identifies a value of $\phi$, indicated in the legend (in degrees).

time average $\mathrm{Nu} \simeq 7.4-7.5$. For these values of $\phi$ the heat transport is no longer strongly intermittent. (3) A higher-Nu regime for $\phi \geqslant 45^{\circ}(\mathrm{Nu} \simeq 10 \pm 0.1)$. For these values of $\phi$, the average values of the Nusselt number converge to a similar quantity, regardless of the axis tilt in this range.

For $\phi=30^{\circ}$, the average value of the Nusselt number for this simulation starts in the high-Nu regime, but then at about $t=0.45$ it shows a decrease, becoming closer to the value observed for $\phi=15^{\circ}$ with $\mathrm{Nu}=7.5$. The meaning of this transition is explored further below. Here we simply note that at large times, the simulation with $\phi=30^{\circ}$ leads to a behavior similar to that observed for $\phi=15^{\circ}$. For all values of $\phi$, the highest vertical heat transport achieved with rotation $(\mathrm{Nu} \simeq 10)$ is significantly less than that achieved without rotation with this setup ( $\mathrm{Nu} \simeq 26$, as reported in Ref. [7]).

To investigate the processes by which convective heat transport varies as $\phi$ is changed, we employ two-dimensional sections of the temperature and velocity fields. Instantaneous horizontal and vertical sections of the temperature fields are displayed in Fig. 2, computed at the later nondimensional time $t=0.75$, when both the $\phi=15^{\circ}$ and $\phi=30^{\circ}$ cases display a lower and stationary average value of $\mathrm{Nu}$. From left to right, the first column shows horizontal sections at mid-depth, while the second and third columns show vertical sections in the $x-z$. plane at $y=\pi$ and in the $y-z$ plane at $x=\pi$, respectively. Because the domain is horizontally periodic, the $x$ and $y$ locations of the vertical sections can be arbitrarily chosen. The orientation of the rotation axis is represented by the solid line in the $y-z$ plane. The same sections are also shown for the $u$ velocity component (along the $x$ direction) in Fig. 3, and for the $v$ velocity component (in the $y$ direction) in Fig. 4. The velocity fields are time averaged over 0.025 thermal times centered on $t=0.75$.

At $\phi=0^{\circ}$ we find a strong, vertically sheared wind in the $x$ direction, homogeneous (i.e., with wave number zero and no modulation) in both the $x$ and $y$ directions at each height $z$, in keeping with the windy convection regime previously described in Ref. [7]. This shear can be clearly seen in the vertical sections of the $y-z$ and $x-z$ planes of the $x$ component of the velocity field $u$, shown in Fig. 3, where the time average 


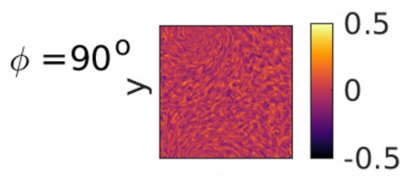

$\mathrm{X}$

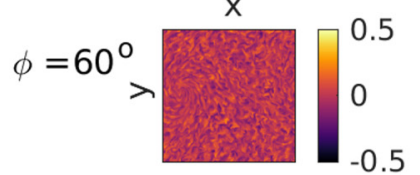

$\mathrm{X}$

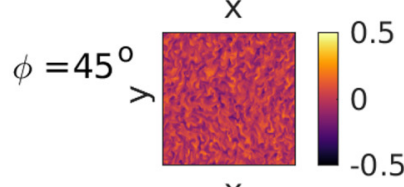

$\mathrm{X}$

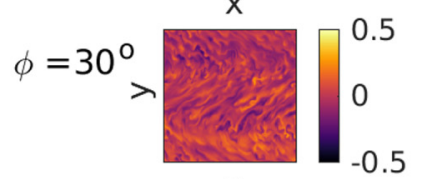

X
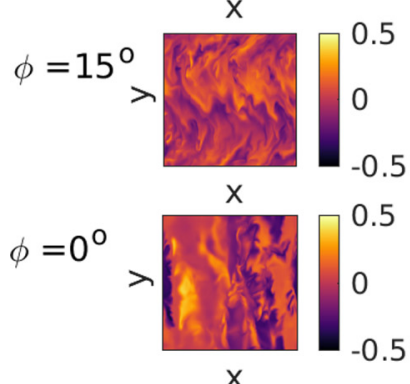
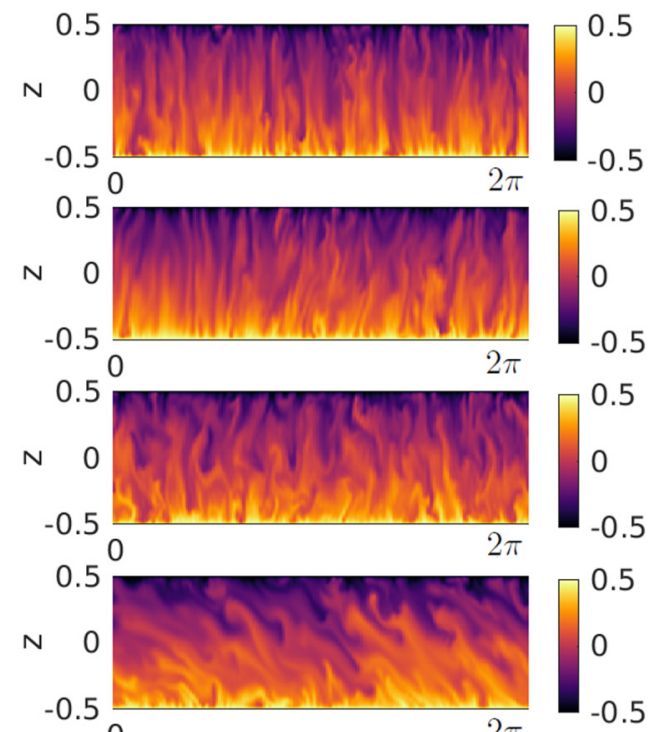$$
\begin{array}{r}
0.5 \\
\mathrm{~N} \\
0 \\
-0.5
\end{array}
$$
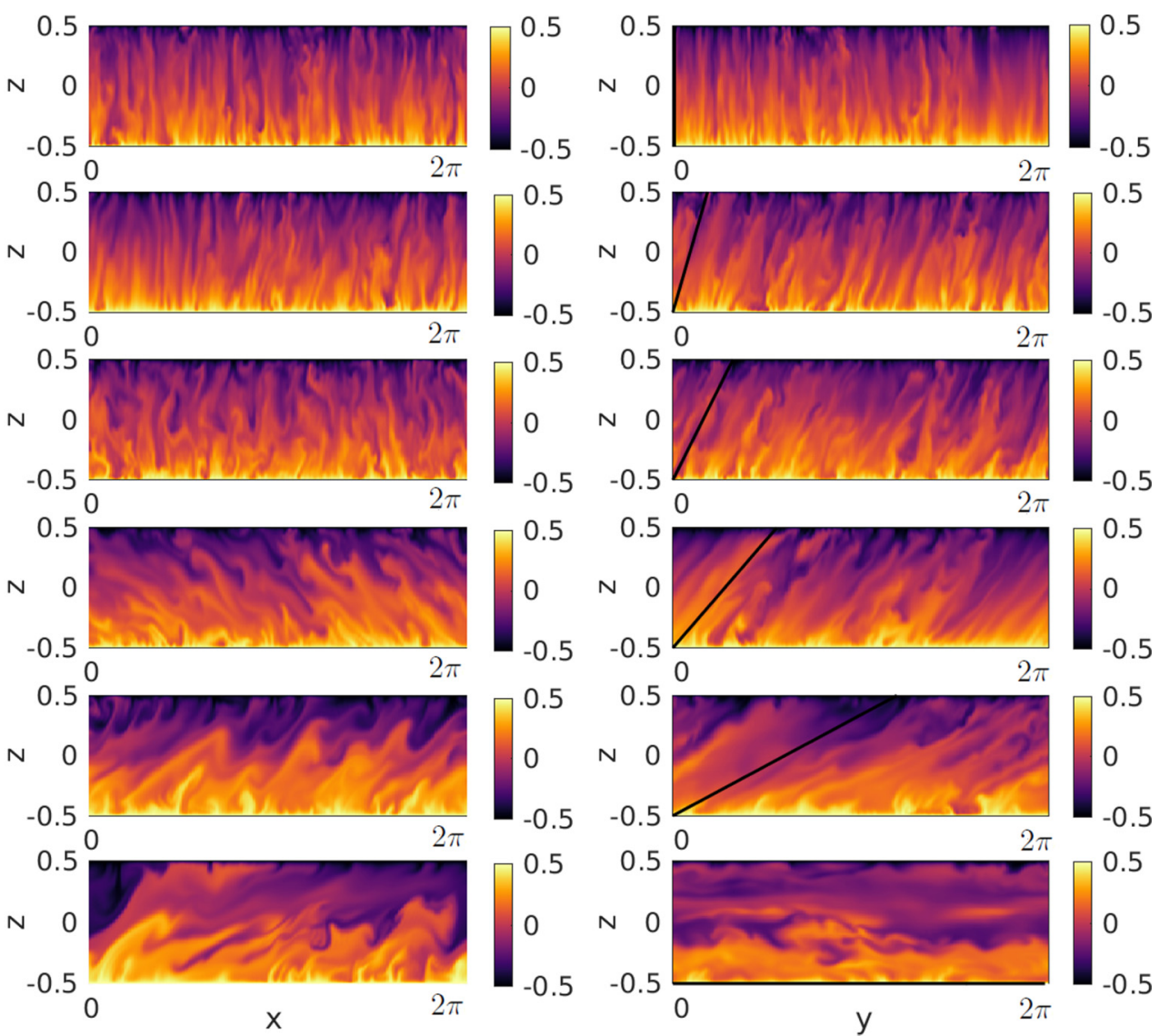

FIG. 2. Horizontal and vertical sections of the temperature field computed at $t=0.75$ for different values of $\phi$, increasing upward. Horizontal sections (leftmost column) are taken at mid-depth, vertical sections in the $x-z$ and $y-z$ planes are computed at $y=\pi$ and at $x=\pi$, respectively. The rotation axis lies in the $y-z$ plane (rightmost column) and its orientation is shown by the solid line. Rising hot plumes are shown in yellow (light gray), and falling cold plumes are shown in dark purple (dark gray).

covers a quiescent portion of the Nusselt intermittency for $\phi=0^{\circ}$. The figure shows strong positive and negative values of $u$ located close to the upper and lower boundaries, respectively. The wind in the transverse $y$ direction is extremely weak, as shown in Fig. 4, indicating an approximately twodimensional flow similar to that studied in Ref. [38]. However, this pattern no longer persists as the latitude increases to $\phi \geqslant 15^{\circ}$. Here, one can still see a strong vertical shear, but the flow is no longer homogeneous in the $y$ direction. As shown in Fig. 3, the $u$ velocity field arranges itself in organized structures with alternating sign of the horizontal velocity along $y$. Specifically, the convective fluid now displays two bands with opposite sign at each height (i.e., with unit wave number along the $y$ direction). $\mathrm{Up}$ to $\phi=30^{\circ}$, the transverse wind $v$ remains weak, as shown in Fig. 4. At higher values of the angle, $\phi \geqslant 45^{\circ}$, we find the appearance of a different regime, characterized by a stronger transverse wind $v$, organized in bands along the $y$ direction, with amplitude comparable to the $u$ component, as shown by the $x-y$ and $x-z$ sections in Fig. 4 (left and central columns, respectively). This new regime is associated with the formation of a cyclonic vortical structure, whose axis is approximately aligned with the rotation axis (compare also with the following Fig. 5). For all orientations of the rotation axis $\phi>0^{\circ}$, the $y-z$ sections in Fig. 3 suggest the existence of a wave-number-one pattern in the $y$ direction, which exhibits a change of orientation parallel to that of the rotation axis. A possible interpretation, albeit somewhat speculative, may be given as to why the wave-number-zero wind in the $y$ direction disappears when $\phi>0^{\circ}$ : In this case, the alignment of the plumes with the rotation axis, now inclined with respect to the horizontal, forces the plumes to hit the top and bottom boundaries, thus impeding the generation of a truly wave-number-zero wind as observed when $\phi=0^{\circ}$. We conclude that the intermittent windy convection regime observed in Ref. [7] can exist only for an almost-horizontal rotation axis (i.e., close to the equator in our spherical shell analogy). Horizontal temperature sections at $\phi=15^{\circ}$ and $\phi=30^{\circ}$ (left column of Fig. 2) show that vertical plumes are distributed along an organized spatial pattern, with rising hot plumes and falling cold plumes gathered in a regular formation along $y$, with an approximately homogeneous distribution along $x$. The corresponding vertical sections of temperature in the $y-z$ plane suggest that the convective plumes at $\phi \geqslant 15^{\circ}$ exhibit a change of orientation parallel to that of the rotation axis, as clearly visible in the rightmost column of Fig. 2. At low values of $\phi$, plumes are slanted, straightening toward the vertical as the angle increases. The tilt of the thermal plumes in the $x-z$ plane (second column of Fig. 2) depends on the particular value of $y$ at which the slice is taken (here, $y=\pi$ ), varying at different values of $y$ and with time. 


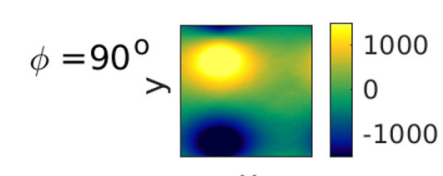

$x$

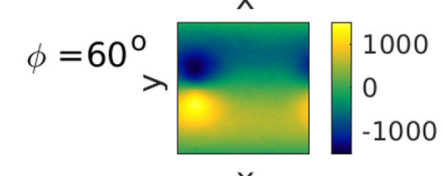

$\mathrm{X}$

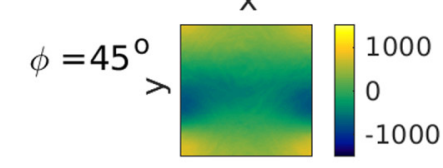

$\mathrm{X}$

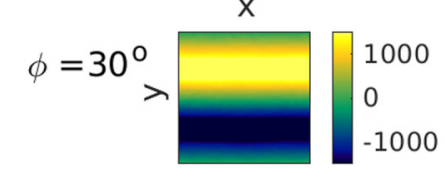

$\mathrm{X}$

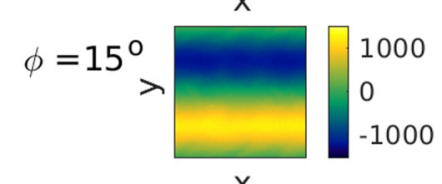

$\mathrm{X}$

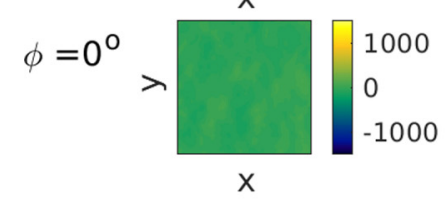

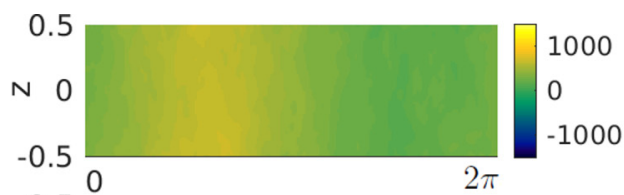
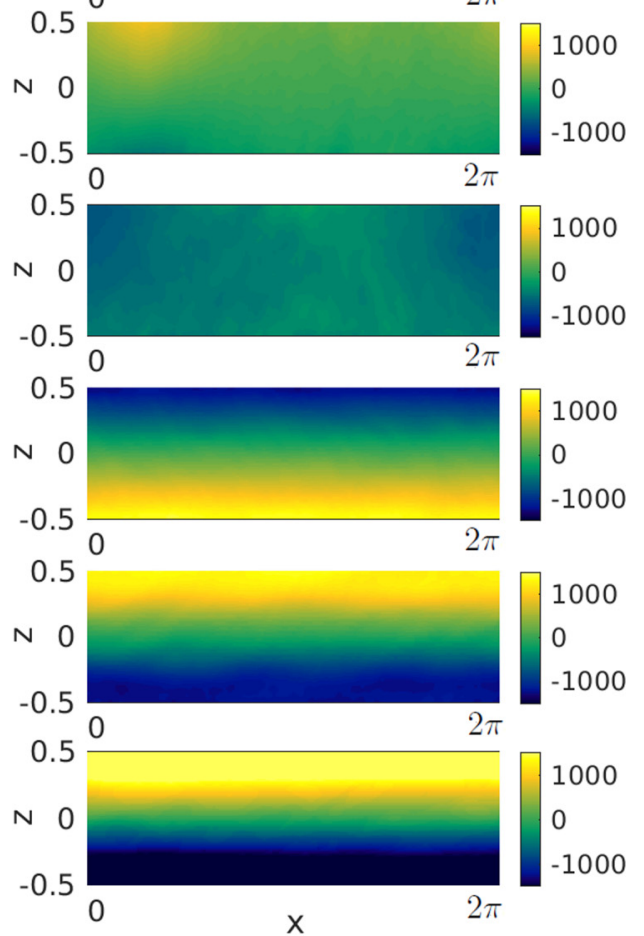

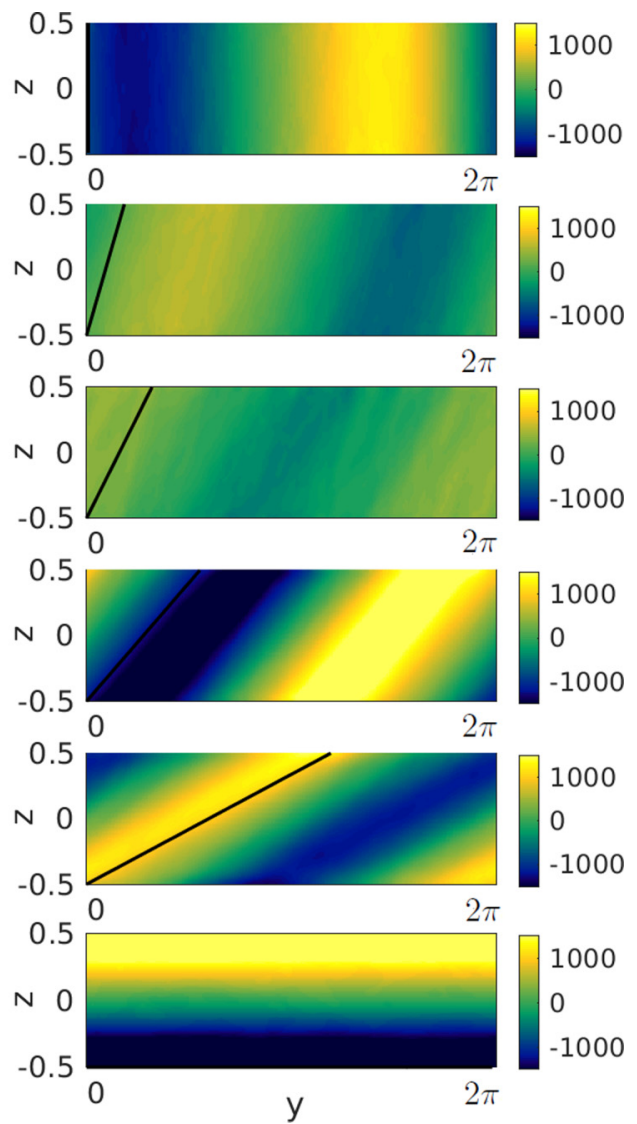

FIG. 3. Horizontal and vertical sections of the $u$ velocity component, averaged over 0.025 thermal times centered on $t=0.75$. Horizontal sections (leftmost column) are taken at mid-depth, and vertical sections in the $x-z$ and $y-z$ planes are computed at $y=\pi$ and at $x=\pi$, respectively. The rotation axis lies in the $y-z$ plane (rightmost column) and is shown as a solid line.

Figure 5 shows the vorticity component along the rotation axis (i.e., $\hat{\mathbf{k}} \cdot \nabla \times \mathbf{u}$ ), time-averaged over 0.025 thermal times centered on $t=0.75$. Three qualitatively different patterns are visible in these fields: (1) a horizontal wave-number-zero field consistent with large-scale windy convection at $\phi=0^{\circ}$; (2) a wave-number-one configuration in the $y$ direction for $\phi=15^{\circ}$ and $\phi=30^{\circ}$; and (3) a large-scale coherent cyclonic vortex at higher values of $\phi$, which becomes vertical for $\phi=90^{\circ}$. The panels in the rightmost column show how the vorticity field aligns with the rotation axis. In the banded regime at lower angles $\phi$, the entire vorticity field is aligned with the rotation axis. Once a large-scale cyclonic vortex forms at larger values of $\phi$, its vorticity also aligns with the rotation axis, as shown by the sections in the $y-z$ plane in Fig. 5.

We quantitatively assess the alignment of the vorticity field with the rotation axis by computing their relative orientation. The vorticity is time averaged over 0.2 thermal times centered on $t=0.75$, and its components along the horizontal and vertical axes are extracted to estimate the angle formed by the vorticity in the $y-z, x-z$, and $x-y$ planes. Table I reports the angles computed for all the latitudes considered. In the low $\phi$ cases, where the wave-number-one solution is reached in the $y-z$ plane, a prevailing orientation can be identified for all points in the domain. For $\phi=90^{\circ}$, the prevailing direction is due to cyclonic and anticyclonic vorticity. In such cases, the prevailing direction is estimated by computing the mode of the distribution of the angles in each plane. Only for the vortex-dominated cases at $\phi=45^{\circ}$ and $60^{\circ}$, due to the broad distribution of angles, can we compute the dominant orientation as the mean orientation of the cyclonic vorticity. The first column reports the values of the rotation axis orientation residing in the $y-z$ plane, while the second column contains the measured prevailing vorticity orientation in the same plane. The third and fourth columns show its value in the $x-z$ and $x-y$ planes, respectively. The values computed confirm a good alignment between the vorticity field and the rotation axis.

The sheared velocity bands observed in Fig. 3 for $\phi \leqslant 30^{\circ}$, tilted along the rotation vector in the $y-z$ plane, are consistent with a strong cyclonic vorticity field aligned with the direction of the rotation axis. At low values of $\phi$, the appearance of a wave-number-one organization of the velocity bands in the $y$ direction allows the vorticity vector to be aligned with the rotation vector even in the absence of a significant transverse $v$ field: For a vorticity vector with a significant vertical component, and in the case of a small transverse velocity $v$, only a strong variation of $u$ along $y$ allows such solution. For larger values of $\phi$, on the other hand, the emergence of a transverse component of the velocity field $v$ allows organization of the flow into a large-scale cyclonic vortex with vorticity tilted along the rotation axis (see also Fig. 5). 


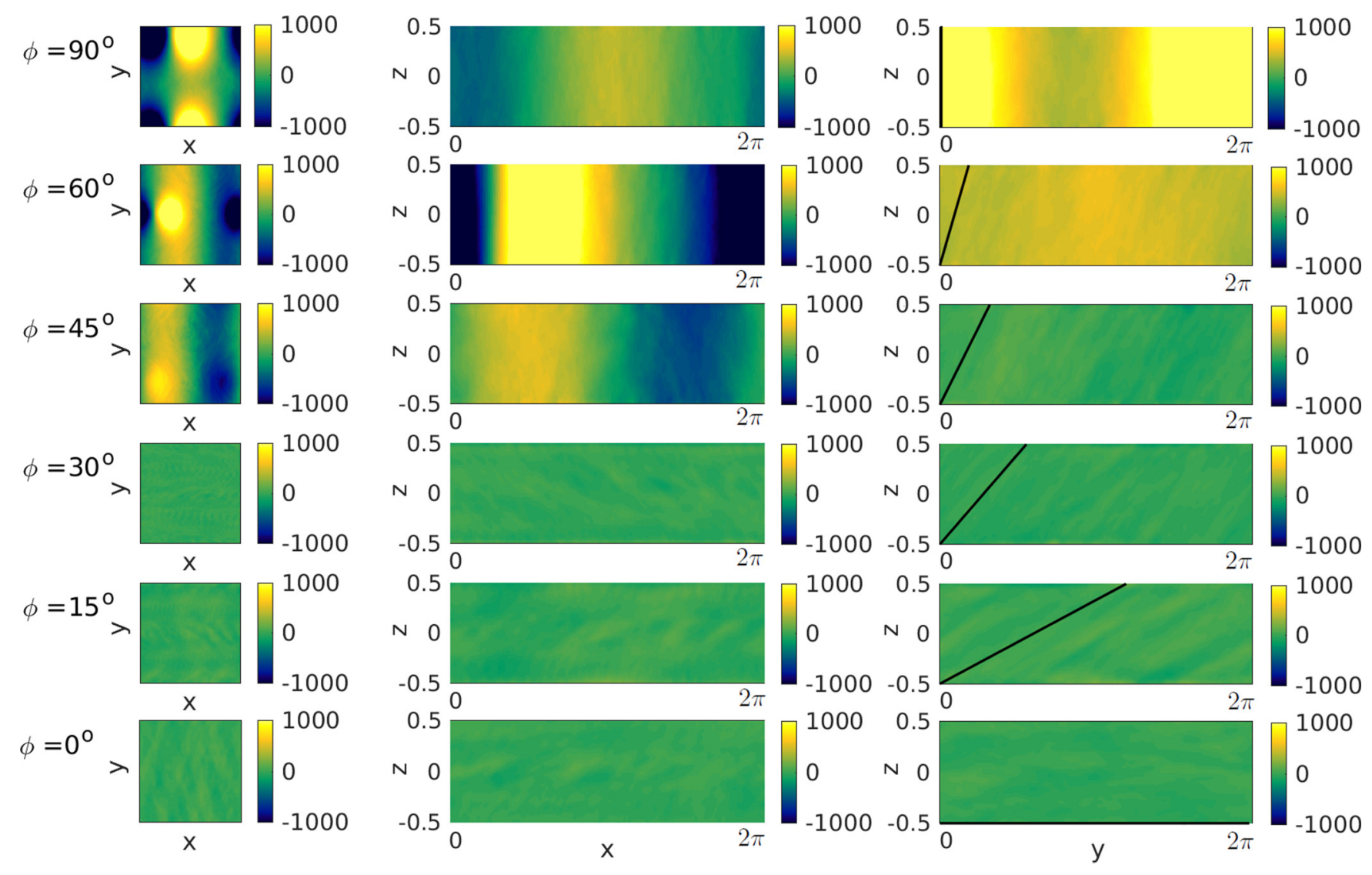

FIG. 4. Horizontal and vertical sections of the $v$ velocity component, averaged over 0.025 thermal times centered on $t=0.75$. Horizontal sections (leftmost column) are taken at mid-depth, and vertical sections in the $x-z$ and $y$ - $z$ planes are computed at $y=\pi$ and at $x=\pi$, respectively. The rotation axis lies in the $y-z$ plane (rightmost column) and is shown as a solid line.

As mentioned above, at $\phi=30^{\circ}$ we observed a transition from a higher- $\mathrm{Nu}$ to a lower- $\mathrm{Nu}$ regime around $t=0.45$. Figure 6 shows horizontal and vertical sections of the temperature and velocity components along $x$ taken at $t=0.3$, before the transition, for both the $\phi=15^{\circ}$ and $\phi=30^{\circ}$ cases. The upper panels show the temperature and the lower panels the velocity fields. For both values of $\phi$, we observe a tilted arrangement of the thermal plumes in the $y-z$ plane. At this time, the velocity field at $\phi=15^{\circ}$ is already organized into a strongly sheared wave-number-one pattern in the $y$ - $z$ plane, while such a configuration is only weakly formed for $\phi=30^{\circ}$, compared to the corresponding section at $t=0.75$ in Fig. 3. The transverse velocities $v$ for $\phi=30^{\circ}$ at this early time are still comparable in amplitude to the $u$ velocities, while, as shown in Fig. 4, they weaken significantly after the transition to the lower-Nusselt-number flow regime. This makes the early-time $\phi=30^{\circ}$ flow more similar to the regime with large-scale vortices observed for $\phi \geqslant 45^{\circ}$ at $t=0.75$ and, in fact, it shares a similar value of the Nusselt number.

We complete this picture by reporting, in Fig. 7, a comparison between the vertical profiles of $u(x, y, z)$ averaged in the $x$ direction, $\langle u\rangle_{x}(y, z)$, at four equally spaced $y$ locations, where $\langle\ldots\rangle_{x}$ indicates an average over the $x$ direction. The figure shows the cases for $\phi=15^{\circ}$ [Figs. 7(a) and 7(c)] and $\phi=30^{\circ}$ [Figs. 7(b) and 7(d)], before (upper row) and after (lower row) the $\phi=30^{\circ}$ transition. Profiles are time averaged over 0.025 thermal times centered on $t=0.3$ and $t=0.75$, respectively.
Before the transition, the $\phi=30^{\circ}$ case is characterized by a much weaker vertical shear at all sections [Fig. 7(b)], with the velocity field not intense enough to sensibly deviate the thermal plumes in the $x-z$ plane (compare with Fig. 6). At the same time, a strongly sheared $u$ profile is instead found at $\phi=15^{\circ}$, as shown in Fig. 7(a). The profile shape depends on the selected value of $y$ but its amplitude does not change with $y$.

As we know from Fig. 1, at this time the strongly sheared flow at $\phi=15^{\circ}$ is characterized by a lower value of $\mathrm{Nu}$ than the weakly sheared $\phi=30^{\circ}$ case. At $t=0.75$, after the transition, the vertical profiles of $\phi=30^{\circ}$ also exhibit a strongly sheared configuration [Fig. 1(d)], in accordance with the formation of the pattern discussed and reported in Figs. 2, 3, and 4. The lower value of $\mathrm{Nu}$ observed at this time for $\phi=30^{\circ}$ is consistent with the stronger deviation that temperature plumes now experience in the $x-z$ plane. The pathway of convective plumes to the opposite boundaries is hindered as the velocity field is organized into strong bands, resulting in a weaker heat transport by convection.

The transition of the $\phi=30^{\circ}$ solution is suggestive of the possible coexistence of multiple solutions for the same parameter set. To address this issue, we explored whether a strongly sheared solution organized in bands (instead of large-scale vortices) could exist also at $\phi \geqslant 45^{\circ}$. To this end, we performed simulations for which the strongly sheared and banded solution for $\phi=30^{\circ}$ at $t=0.9$ was used as the 


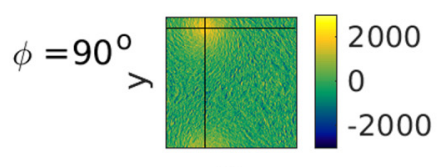

$X$

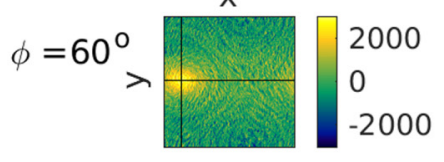

$\mathrm{X}$

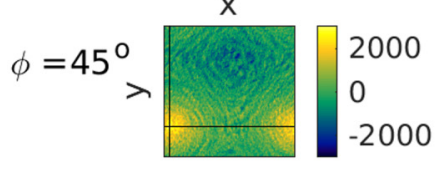

$X$

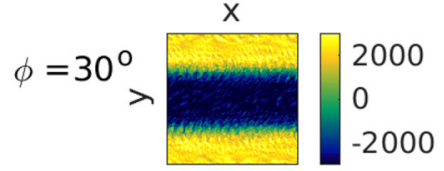

$X$

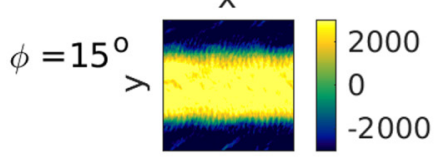

$\mathrm{X}$

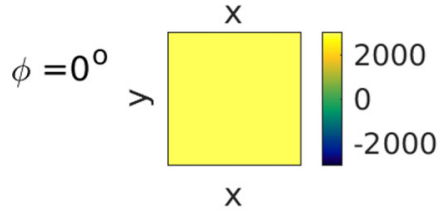

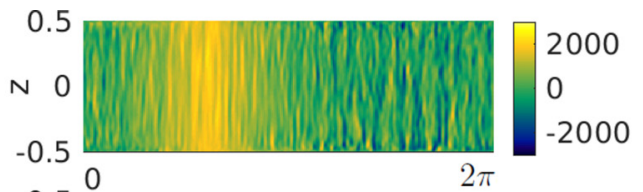
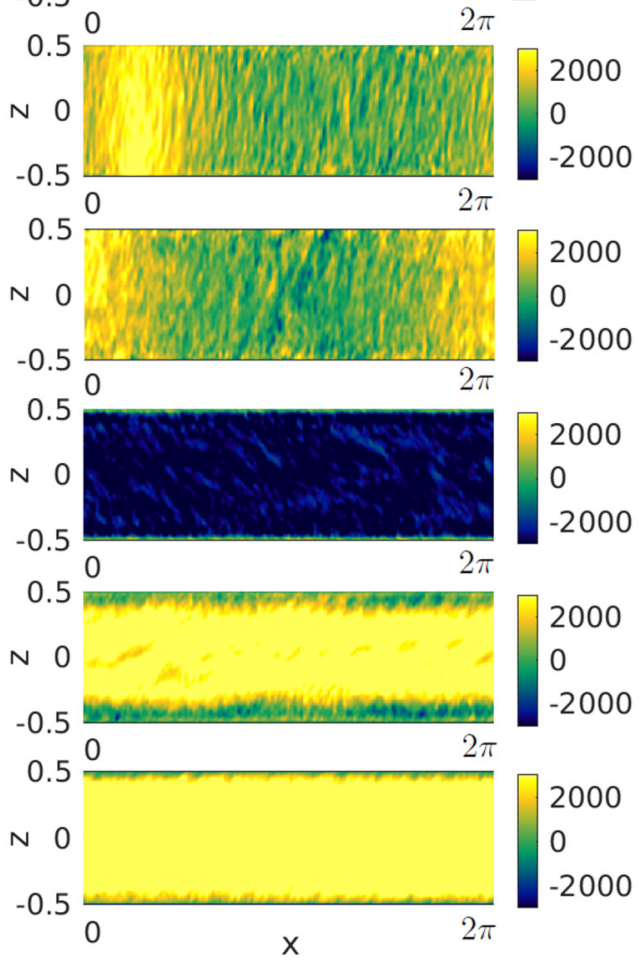
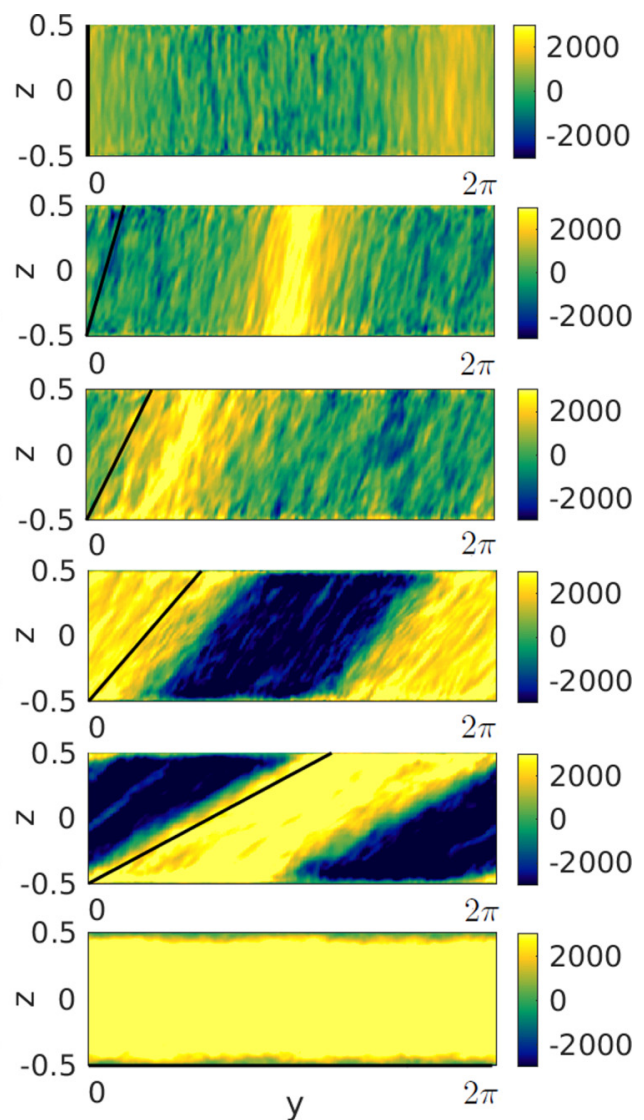

FIG. 5. Horizontal and vertical sections of the vorticity component along the rotation axis, averaged over 0.025 thermal times centered on $t=0.75$. Horizontal sections (leftmost column) are taken at mid-depth, vertical sections in the $x-z$ and $y-z$ planes are computed at $y=\pi$ and at $x=\pi$, respectively, for $\phi \leqslant 30^{\circ}$. For $\phi \geqslant 45^{\circ}$ the sections are taken through the large-scale cyclonic vortex that has formed. The solid lines in the horizontal sections show the positions of the two vertical sections. The rotation axis lies in the $y-z$ plane (rightmost column) and is shown as a solid line.

initial condition for two cases at $\phi=45^{\circ}$ and $\phi=60^{\circ}$. From this restart, the simulations were continued for a further time interval $\Delta t=0.35$. Horizontal and vertical sections for this second solution for $\phi=45^{\circ}$ and $\phi=60^{\circ}$, after $\Delta t=0.15$ integration time, are displayed in Fig. 8, in which temperature, horizontal velocity components, and the vorticity component along the rotation axis are shown. In both cases we find that the initial high-amplitude shear persists at these higher angles with a low transverse velocity $v$. Vorticity aligns with the orientation of the rotation axis, as shown in Table II, where the prevailing angle of vorticity in the $y-z$ plane was computed using the same procedure as for Table I. This confirms that at these intermediate values of $\phi$, (at least) two different solutions coexist. Consistently with the higher shear in these configurations, we find that for these solutions the Nusselt number settles at the lower average values $\mathrm{Nu}=8.3$ $\left(\phi=45^{\circ}\right)$ and $\mathrm{Nu}=9.4\left(\phi=60^{\circ}\right)$ (both averaged for $\Delta t=$ 0.3 , skipping the first 0.05 time units). These values are lower than those attained by the solution with large-scale vortices and reported earlier in Fig. $1(\mathrm{Nu} \simeq 10.0$ and $\mathrm{Nu} \simeq 9.9$, respectively). Note that these values are larger than the values of $\mathrm{Nu}$ achieved at $\phi \leqslant 30^{\circ}$, but this is consistent with the fact that since the angles formed by the velocity bands are higher, the shear experienced by the rising thermal plumes is lower. Such a result also shows the existence of a bistable state for these values of $\phi$, additionally suggesting that an external perturbation or a particularly large turbulent fluctuation could allow the system to slide between the two equilibria.

Finally, we also verified that if a banded solution from $\phi>0^{\circ}$ is used as initial condition for a run at $\phi=0^{\circ}$, the band

TABLE I. The orientation $\phi$ of the rotation axis in the $y-z$ plane is reported in the leftmost column. The second column contains the mode of the vorticity orientation in the same plane, and the third and fourth columns show its value in the $x-y$ and $x-z$ planes, respectively. The quantities $\omega_{x}, \omega_{y}$, and $\omega_{z}$ denote the components of the vorticity in the $x, y$, and $z$ directions, respectively. In the cases of $\phi=45^{\circ}$ and $\phi=60^{\circ}$, the orientation of the large-scale vortex is computed as the mean orientation of the cyclonic vorticity.

\begin{tabular}{lccc}
\hline \hline$\phi$ & $\arctan \left(\omega_{z} / \omega_{y}\right)$ & $\arctan \left(\omega_{x} / \omega_{y}\right)$ & $\arctan \left(\omega_{x} / \omega_{z}\right)$ \\
\hline $0^{\circ}$ & 0.00 & $-0.41^{\circ}$ & $-89.94^{\circ}$ \\
$15^{\circ}$ & 14.94 & $-2.18^{\circ}$ & $-3.09^{\circ}$ \\
$30^{\circ}$ & 30.03 & $-1.11^{\circ}$ & $0.27^{\circ}$ \\
$45^{\circ}$ (vortex) & 44.17 & $12.12^{\circ}$ & $-2.66^{\circ}$ \\
$60^{\circ}$ (vortex) & 54.31 & $9.75^{\circ}$ & $-4.55^{\circ}$ \\
$90^{\circ}$ (vortex) & \pm 89.7 & $-4.35^{\circ}$ & $0.01^{\circ}$ \\
\hline \hline
\end{tabular}



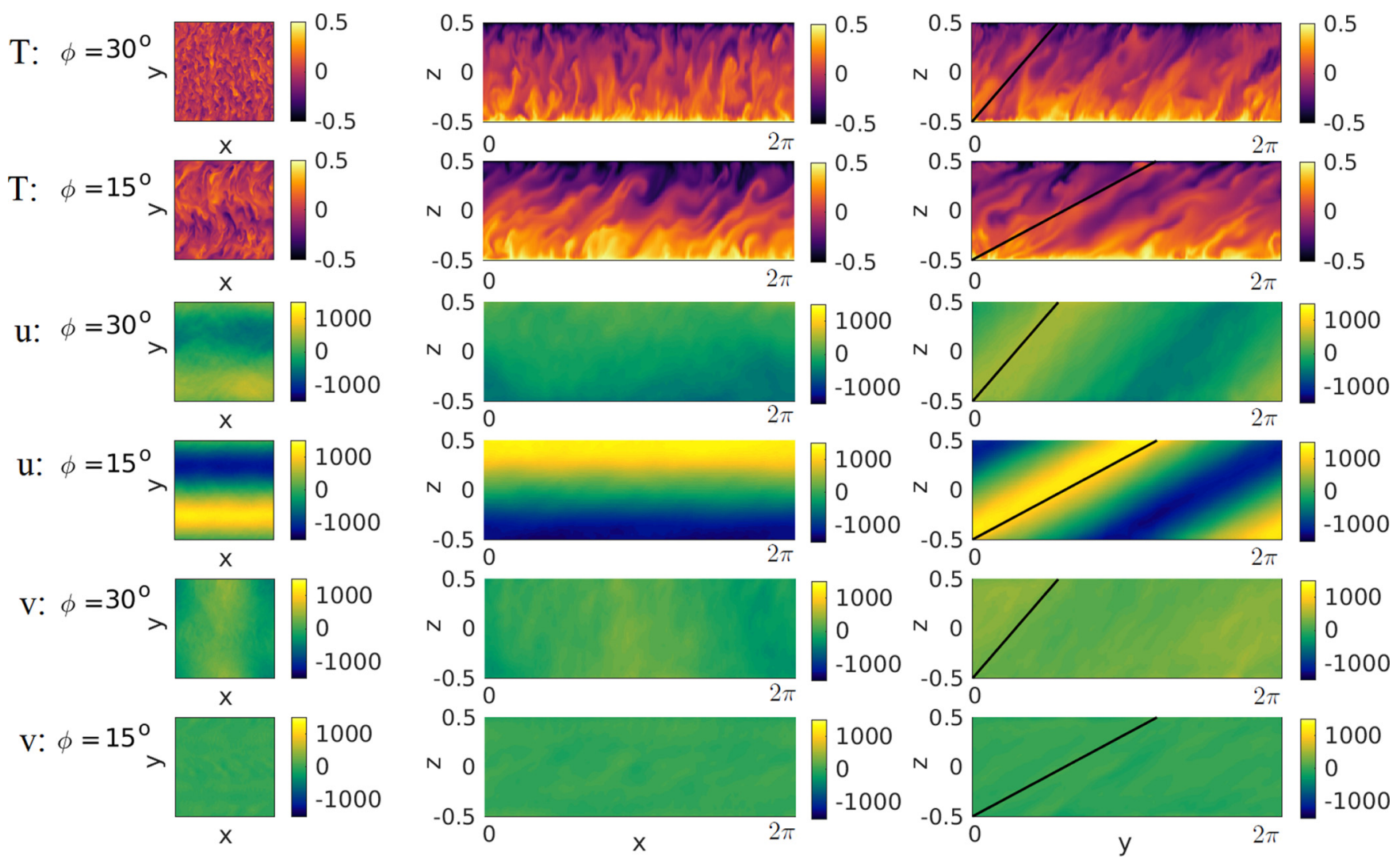

FIG. 6. Horizontal and vertical sections of the temperature and velocity components along the $x$ direction at $\phi=15^{\circ}$ and $\phi=30^{\circ}$ at $t=0.3$. From top to bottom: temperature sections for $\phi=30^{\circ}$ and $\phi=15^{\circ} ; u$ velocity sections at $\phi=30^{\circ}$ and $\phi=15^{\circ} ; v$ velocity sections at $\phi=30^{\circ}$ and $\phi=15^{\circ}$. Velocity sections are time averaged over 0.025 thermal times and centered on $t=0.3$. Horizontal sections are taken at mid-depth, and vertical sections in the $x-z$ and $y-z$ planes are computed at $y=\pi$ and at $x=\pi$, respectively. The rotation axis lies in the $y-z$ plane (rightmost column) and is shown as a solid line.

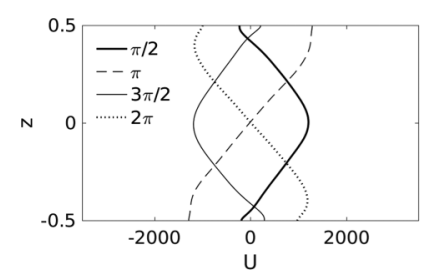

(a)

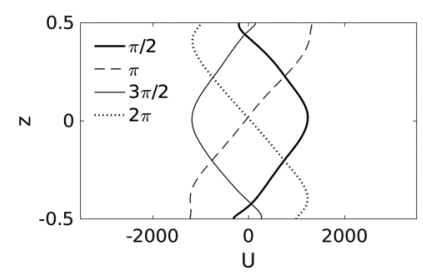

(c)

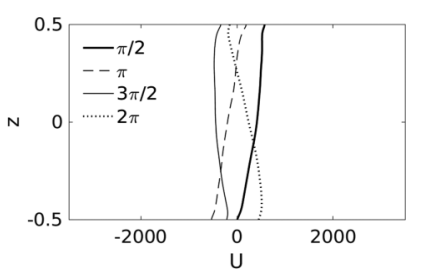

(b)

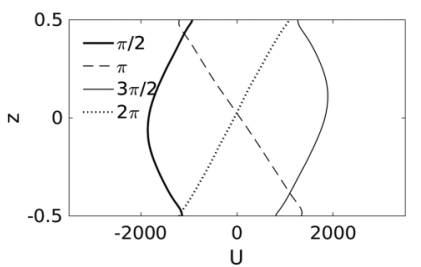

(d)

FIG. 7. Vertical profiles of the $u$ velocity component averaged along the $x$ direction and time averaged over 0.025 thermal times centered on $t=0.3[$ [a) and (b)] and $t=0.75[(\mathrm{c})$ and (d)] for $\phi=15^{\circ}$ [left column, panels (a) and (c)] and $\phi=30^{\circ}$ [right column, panels (b) and (d)]. Profiles are computed at four $y$ locations: $y=$ $\pi / 2, y=\pi, y=3 \pi / 2, y=2 \pi$. Profiles at $\phi=15^{\circ}$ exhibit the same shape at $t=0.3$ (a) and $t=0.75$ (c) since no intermediate transition occurs at this angle, unlike for the $\phi=30^{\circ}$ case, in which the lower value of the Nusselt number at $t=0.75$ is associated with the change in the profiles (d).

structure in the $y$ direction disappears and the intermittent windy convection solution is recovered.

\section{CONCLUSIONS AND PERSPECTIVES}

In this work we have explored how rapidly rotating convection is affected by the orientation of the rotation axis for a model geometry and setup already employed in previous works $[7,35,36]$. We reproduced the windy intermittent convection observed in Ref. [7] for a system with background rotation perpendicular to the gravity direction (the case $\phi=$ $0^{\circ}$ ), as well as the generation of large-scale vortices observed in Refs. $[6,22]$, when the rotation and gravity vectors are parallel $\left(\phi=90^{\circ}\right)$. The relationship between thermal and dynamical times involved in this problem is governed by a factor $\sqrt{\sigma \mathrm{Ra}}$, making the simulations long enough to cover at least the main features of the convective dynamics.

In general, we found that the convective plumes tend to align with the direction of the rotation axis in the plane where the axis resides (the $y-z$ plane in our case). When $\phi=0^{\circ}$, this tendency conflicts with the need for transporting heat along the vertical, and an intermittent, large-scale wind is generated, with weak variations in the horizontal. When $\phi>0^{\circ}$, this intermittent behavior is found to disappear.

We also verified the observation that the vorticity vector tends to align with the rotation axis. When $\phi=0^{\circ}$, this 

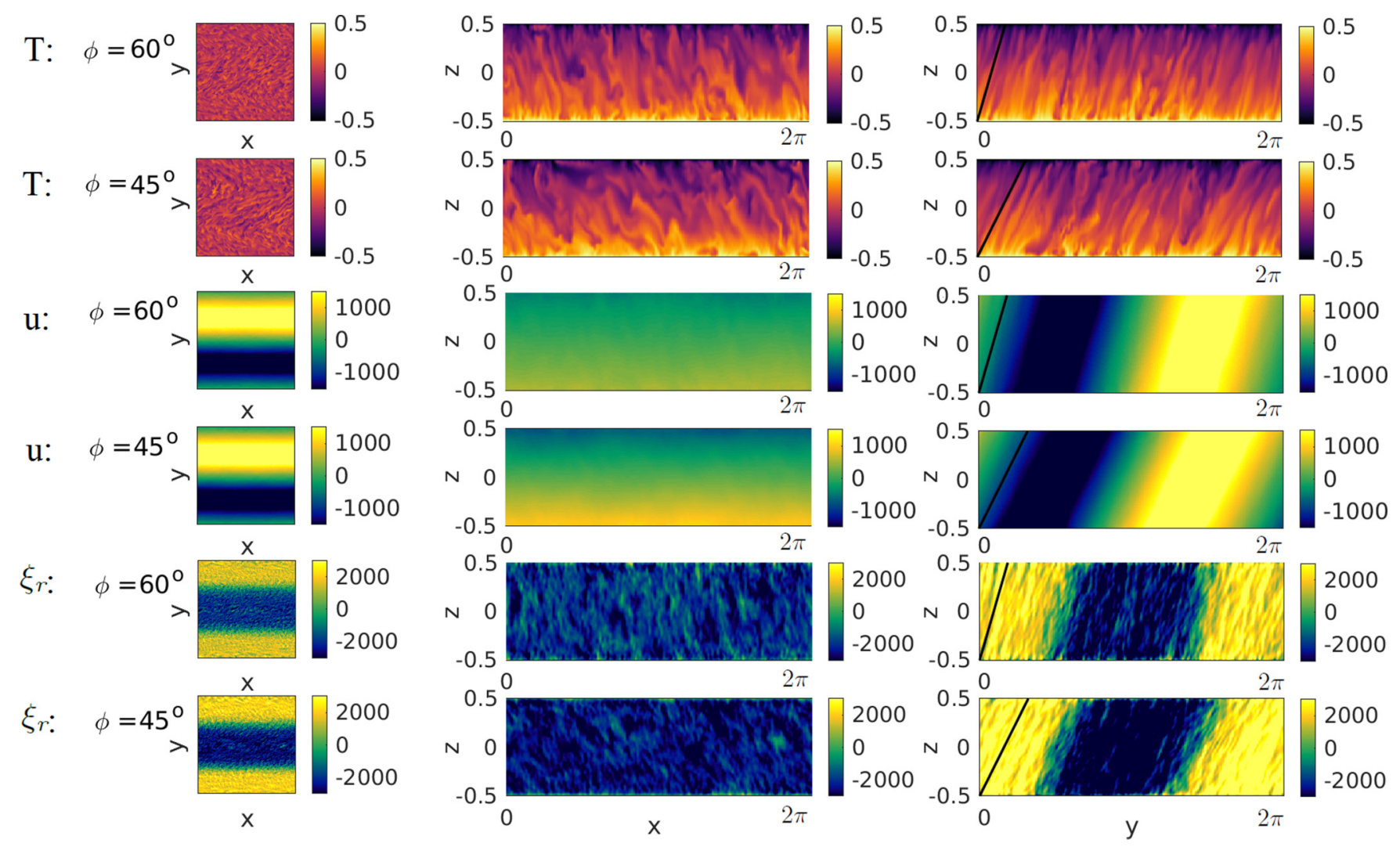

FIG. 8. Horizontal and vertical sections for the simulations at $\phi=45^{\circ}$ and $\phi=60^{\circ}$ restarted from the solution at $\phi=30^{\circ}$ at $t=0.9$, taken after $\Delta t=0.25$. The panels show temperature (upper two rows, denoted as $T$ ), velocity component along the $x$ direction (middle two rows, denoted as $u$ ) and vorticity component along the rotation axis (bottom two rows, denoted as $\xi_{r}$ ). The velocity component along the $y$ direction is very weak and their sections are thus omitted. The velocity and vorticity fields are time averaged over 0.025 thermal times. The solid line in the rightmost column indicates the direction of the rotation axis. Horizontal sections (leftmost column) are taken at mid-depth, and vertical sections in the $x-z$ and $y-z$ planes are computed at $y=\pi$ and at $x=\pi$, respectively

is satisfied already by a horizontal velocity field in the $x$ direction, homogeneous in both horizontal directions and without significant transverse velocity $v$. The intermittent windy convection configuration, with zero wave number in both horizontal directions, disappears as soon as $\phi>0^{\circ}$. In this case the vorticity vector acquires a vertical component, something that can be achieved only through a variation of the velocity $u$ in the $y$ direction or the appearance of a comparable $v$ velocity varying in $x$.

Consequently, at low but finite values of $\phi$, the system dynamics leads to the formation of a $u$ field organized in positive and negative bands in the $y$ direction, with unit wave number, still without a significant transverse $v$

TABLE II. Vorticity components for the restarted solutions. The angle $\phi$ is reported in the leftmost column. The second column contains the mode of the vorticity orientation in the $y-z$ plane, and the third and fourth columns show the vorticity orientation in the $x-y$ and $x-z$ planes, respectively.

\begin{tabular}{lccc}
\hline \hline$\phi$ & $\arctan \left(\omega_{z} / \omega_{y}\right)$ & $\arctan \left(\omega_{x} / \omega_{y}\right)$ & $\arctan \left(\omega_{x} / \omega_{z}\right)$ \\
\hline $45^{\circ}$ & 44.83 & $-0.20^{\circ}$ & $1.71^{\circ}$ \\
$60^{\circ}$ & 59.85 & $-0.02^{\circ}$ & $2.46^{\circ}$ \\
\hline \hline
\end{tabular}

velocity. Through appropriately initialized experiments, we have verified that this solution also exists up to $\phi=60^{\circ}$. For intermediate values of $\phi$, this solution coexists with a different regime, in which a robust transverse velocity $v$, varying along $x$, appears. This solution is associated with the formation of large-scale vortical structures, as reported in Ref. [22] for the case with vertical rotation axis. Furthermore, these large-scale vortices are found to be tilted along the rotation vector.

In the strongly sheared flow regime, the global vertical heat flux enhancement by means of convection, as measured by the Nusselt number, is shown to be significantly weakened. Thermal plumes are deviated by the shear before reaching the opposite boundary, disrupting heat transport pathways, as discussed in Ref. [7]. In the regime where large-scale longlived vortices appear, vertical transport is higher but is still affected by rotation and is significantly reduced compared to the nonrotating case, as discussed in Ref. [6].

At the moment, we cannot say whether increasing the horizontal extent of the domain will lead to larger and larger structures, keeping a wave-number-one structure, or if solutions with a different wave number in the $y$ direction appear. This issue will be explored in future works, together with a wider exploration of parameter space (Rayleigh number, Prandtl number, rotation velocity) and a more complete examination of the range of existence of the different solutions. 
We conclude by noting that the configuration adopted here, albeit extremely simplified, can be conceptually useful in understanding convective motions in rapidly rotating planetary flows. In particular, the results reported bear intriguing relationships to the recent observations of Jupiter, which show strong jets at low-to-mid latitudes (low $\phi$ ) and well-defined intense vortices at high latitudes (large $\phi$ ) [18]. Interestingly, at moderate latitudes our results suggest the possible coexistence of jets and tilted-vortex solutions. Further work is clearly needed to unravel completely the possible similarities between the simplified configuration adopted here and the complex dynamics of turbulent convection in spherical shells.

\section{ACKNOWLEDGMENTS}

The research reported in this work was partially supported by OGS and CINECA under the HPC-TRES Program Award No. 2015-06 and by ASI-INAF Agreement No. 2018-25-HH.0 "Scientific activities for JUICE phase C/D." We acknowledge CINECA for providing computing resources through ISCRA Grants No. IsC43_STARWAR-, No. IsC54_SIPRACo, and No. IsC62_SIPRACo2. We are grateful to S. Tobias for useful comments on this work and to D. Goluskin for useful discussions on rotating convection. Preliminary discussions on this work took place while D.W.H. was supported by a Royal Society International Exchange grant. E.A.S. contributed to the research but could not contribute to the writing.
[1] E. D. Siggia, Ann. Rev. Fluid Mech. 26, 137 (1994).

[2] G. Ahlers, S. Grossmann, and D. Lohse, Rev. Mod. Phys. 81, 503 (2009).

[3] D. Avsec, Tourbillons Thermoconvectifs Dans l'Air. Application à la Météorologie, Ph.D. thesis, Université de Paris (1939).

[4] S. Chandrasekhar, Hydrodynamic and Hydromagnetic Stability (Clarendon Press, Oxford, 1961).

[5] K. Julien, S. Legg, J. C. McWilliams, and J. Werne, J. Fluid Mech. 322, 243 (1996).

[6] A. B. Pieri, F. Falasca, J. V. Hardenberg, and A. Provenzale, Phys. Lett. A 380, 1363 (2016).

[7] J. von Hardenberg, D. Goluskin, A. Provenzale, and E. A. Spiegel, Phys. Rev. Lett. 115, 134501 (2015).

[8] R. Barkan, K. B. Winters, and S. G. Llewellyn Smith, J. Fluid Mech. 723, 556 (2013).

[9] Y. Nakagawa and P. Frenzen, Tellus 7, 1 (1955).

[10] H. T. Rossby, J. Fluid Mech. 36, 309 (1969).

[11] Y. Hu, R. E. Ecke, and G. Ahlers, Phys. Rev. Lett. 74, 5040 (1995).

[12] S. Weiss and G. Ahlers, J. Fluid Mech. 684, 407 (2011).

[13] K. L. Chan, Astron. Nachr. 328, 1059 (2007).

[14] K. L. Chan and H. G. Mayr, Earth Planet. Sci. Lett. 371-372, 212 (2013).

[15] J.-Q. Zhong, R. J. A. M. Stevens, H. J. H. Clercx, R. Verzicco, D. Lohse, and G. Ahlers, Phys. Rev. Lett. 102, 044502 (2009).

[16] S. Weiss, R. J. A. M. Stevens, J.-Q. Zhong, H. J. H. Clercx, D. Lohse, and G. Ahlers, Phys. Rev. Lett. 105, 224501 (2010).

[17] I. Grooms, K. Julien, J. B. Weiss, and E. Knobloch, Phys. Rev. Lett. 104, 224501 (2010).

[18] A. Adriani, A. Mura, G. Orton, C. Hansen, F. Altieri, M. L. Moriconi, J. Rogers, G. Eichstädt, T. Momary, A. P. Ingersoll, G. Filacchione, G. Sindoni, F. Tabataba-Vakili, B. M. Dinelli,
F. Fabiano, S. J. Bolton, J. E. P. Connerney, S. K. Atreya, J. I. Lunine, F. Tosi et al., Nature 555, 216 (2018).

[19] J. Pedlosky, Geophysical Fluid Dynamics (Springer, Berlin, 1987).

[20] F. H. Busse, J. Fluid Mech. 44, 441 (1970).

[21] F. H. Busse, Phys. Fluids 14, 1301 (2002).

[22] C. Guervilly, D. W. Hughes, and C. A. Jones, J. Fluid Mech. 758, 407 (2014).

[23] B. Favier, L. J. Silvers, and M. R. E. Proctor, Phys. Fluids 26, 096605 (2014).

[24] K. Julien, A. M. Rubio, I. Grooms, and E. Knobloch, Geophys. Astrophys. Fluid Dyn. 106, 392 (2012).

[25] U. R. Christensen, J. Fluid Mech. 470, 115 (2002).

[26] M. Heimpel and J. Aurnou, Icarus 187, 540 (2007).

[27] Y. Kaspi, G. R. Flierl, and A. P. Showman, Icarus 202, 525 (2009).

[28] F. H. Busse, Chaos 4, 123 (1994).

[29] K. Zhang and X. Liao, Theory and Modeling of Rotating Fluids (Cambridge University Press, Cambridge, 2017).

[30] M. Heimpel, J. Aurnou, and J. Wicht, Nature 438, 193 (2005).

[31] D. H. Hathaway, J. Toomre, and P. A. Gilman, Geophys. Astrophys. Fluid Dyn. 15, 7 (1980).

[32] L. K. Currie, The Driving of Mean Flows by Convection, Ph.D. thesis, University of Leeds (2014).

[33] D. H. Hathaway and R. C. J. Sormerville, J. Fluid. Mech. 126, 75 (1983).

[34] Lord Rayleigh, Phil. Mag. 32, 529 (1916).

[35] A. Parodi, J. von Hardenberg, G. Passoni, A. Provenzale, and E. A. Spiegel, Phys. Rev. Lett. 92, 194503 (2004).

[36] J. von Hardenberg, A. Parodi, G. Passoni, A. Provenzale, and E. A. Spiegel, Phys. Lett. A 372, 2223 (2008).

[37] G. Passoni, G. Alfonsi, and M. Galbiati, Int. J. Numer. Methods Fl. 38, 1069 (2002).

[38] D. Goluskin, H. Johnston, G. R. Flierl, and E. A. Spiegel, J. Fluid Mech. 759, 360 (2014). 